\title{
A Compact Tri-Band Bandpass Filter with High Out-of-Band Rejection
}

\author{
Mohamad Farhat \\ School of Engineering \\ The Australian College of \\ Kuwait, Kuwait \\ m.farhat@ack.edu.kw
}

\author{
Mohammed Abdul-Niby \\ School of Engineering \\ The Australian College of \\ Kuwait, Kuwait \\ m.nibi@ack.edu.kw
}

\author{
Michel Nahas \\ School of Engineering \\ The Australian College of \\ Kuwait, Kuwait \\ m.nahas@ack.edu.kw
}

\author{
Majdi Alomari \\ School of Engineering \\ The Australian College of \\ Kuwait, Kuwait \\ m.alomari@ack.edu.kw
}

\begin{abstract}
This paper presents a planar tri-band bandpass filter with high out-of-band rejection over a wide band. The filter is based on two pairs of $\lambda / 4$ resonators embedded inside an open loop ring resonator without any size increase, where each pair of resonators are electromagnetically coupled to each other and the feedlines. This results in the excitations of passbands, where the first passband is generated by the open loop resonators. The second and the third passbands are excited by $\lambda / 4$ resonators. The proposed technique provides sufficient degrees of freedom to control the center frequency and bandwidth of the three passbands independently. In addition, the six transmission zeros created around the passbands results in a tri-band filter with high selectivity, sharp $3 \mathrm{~dB}$ cut-off frequency, high isolation, low passband insertion-loss and high out-of-band harmonic rejection across an ultra-broadband frequency range up to $17 \mathrm{GHz}$. The proposed technique has the ability to switch from triple to dual band by removing one pair of the inner resonators. Design methodology and simulation results of the filter are provided.
\end{abstract}

Keywords-tri-band; bandpass; filter; microstrip filter; planer

\section{INTRODUCTION}

The growing demand of wireless communication applications necessitates RF transceivers to operate in multiple but separated frequency bands so that users can access various services, such as WLAN, IEEE 802.16 (WiMAX) and RFID, with a single multimode handset or terminal. Higher order bandpass filters are routinely constituted by cascading multiple single-/dual-mode resonators. This straightforward approach unfortunately increases the overall filter size and raises the fabrication cost to a great extent. Hence there is a demand for tri-band filters that are small enough to be easily integrated inside RF transceivers. Other salient characteristics sought after in tri-band filters are low insertion-loss, excellent passband selectivity in order to efficiently utilize the EM spectrum, and wide stopband or out-of-band rejection to minimize EMI. These requirements present a challenge for the design engineers.

Various design approaches and structures have been used to develop tri-band BPFs. In [1], the tri-band filter was realized by cascaded multiband resonators. However, the design parameters were too complex and the circuit size is relatively high. To reduce the circuit size, many tri-band filters have been realized with a stepped impedance resonator (SIR) because of their multiband behavior [2-5]. In [2], a tri-band bandpass filter was designed using a combined quarter-wavelength resonator. A trisection SIR [3, 4] could be used to design the tri-band filter, and three passbands at any desired frequencies can be obtained by properly determining the impedance ratio. A multimode stepped-impedance resonator with a $0 \circ$ tapped-feed structure was proposed to design a miniaturized tri-band BPF in [5]. In [3], to provide more freedom to control the filter harmonics responses, tri-section stepped-impedance resonators (TSSIRs) were proposed to build up tri-band BPFs. However, the resonant frequencies of the SIR are dependent, complicating the filter design. Modified stub-loaded resonator [6-8] have been utilized to design tri-band BPFs. In [6], the authors proposed a series of multi-stub loaded resonators to design tri-band BPFs. Moreover, even and odd-mode analysis is utilized twice to analyze the resonance characteristics of multi-stub loaded resonator. In [7], a tri-band filter was designed by one set of stub-loaded shortended resonators. Three embedded bending stub resonators were employed to achieve tri-band filter with compact size [8]. Although the resulting filters are miniature in size, but have a high insertion-loss, the $3 \mathrm{~dB}$ roll-off is skirt not sharp, and low out-of-band rejection.

In this paper, a novel tri-band bandpass filter is presented that comprises of the main open loop resonators in which are embedded two pairs of $\lambda / 4$ resonators. Each pair of the embedded resonators, is designed to operate at a different frequency and generates three passbands without increasing the size of the main open loop resonators. The first passband is determined by the length of the open loop resonators, the second passband is determined by resonators 3 and 4, while the third passband is determined by resonators 5 and 6 as shown in Figure 1. The proposed tri-band filter generates six transmission zeros located around the passbands to realize high selectivity, sharp 3 $\mathrm{dB}$ roll-off and high out-of-band rejection. The planar structure facilitates the design and reduces fabrication cost. The filter is miniature compared to the aforementioned filters with a size of $0.25 \lambda_{\mathrm{g}} \times 0.3 \lambda_{\mathrm{g}}$, which eases integration in wireless systems. The three pairs of resonators provide sufficient degree of freedom to control the center frequency and bandwidth of the three passband responses. Based on the proposed concept, the filter was designed and simulated at 3/6.9/8.6 GHz. 


\section{FILTER CONFIGURATION AND ANALYSIS}

\section{A. Resonators Configuration}

Figure 1 shows the topology of the microstrip bandpass filter. This is a second order filter having an asymmetric structure. The filter consists of two open loop resonators in a Cshape configuration as shown in Figure 1(a), which normally exhibits a single passband response with two transmission zeros to generate a quasi-elliptical response. In the example shown in Figure 2, the passband of such a resonant structure has a centre frequency of $3.5 \mathrm{GHz}$.

The lower transmission zero $\left(f_{\mathrm{L}}\right)$ depends on the length L2 and its location can be calculated using (1); and the upper transmission zero $\left(f_{\mathrm{U}}\right)$ depends on $\mathrm{L} 1$ and its location can be calculated using (2) [9]. Therefore the centre frequency of the first passband $\left(f_{1}\right)$ can be calculated using (3). Two $50 \Omega$ lines connected to the resonators acts as input/output ports with width $\mathrm{W} 1$. The main $\mathrm{C}$-shape resonator structure was modified with the inclusion of four $\lambda / 4$ resonators embedded inside the open loop resonators, as shown in Figure 1(b). One end of these embedded resonators is shorted to ground. The resulting topology represents the proposed microstrip tri-band bandpass filter.

The embedded resonators 3 and 4 generates the second passband with a centre frequency $f 2$ which is determined by the length L3 using Eqn. (4); and resonators 5 and 6 generates the third passband with a centre frequency $f 3$ determined by length L4 using Eqn.(5), the three passbands are shown in Figure 2.

$$
\begin{gathered}
f_{L}=\frac{n c}{4 L_{2} \sqrt{\varepsilon_{e f f}}} \\
f_{U}=\frac{n c}{4 L_{1} \sqrt{\varepsilon_{e f f}}} \\
f_{1}=\frac{f_{U}-f_{L}}{2} \\
f_{2}=\frac{n c}{4 L_{3} \sqrt{\varepsilon_{e f f}}} \\
f_{4}=\frac{n c}{4 L_{4} \sqrt{\varepsilon_{e f f}}}
\end{gathered}
$$

Where $\varepsilon_{e f f}$ is the effective dielectric constant, $n$ is the mode number $(n=1,3,5 \ldots$,$) and c$ is the speed of light in free space $\left(3 \times 10^{8} \mathrm{~m} / \mathrm{s}\right)$.

The open loop C-shape resonators have a low out-of-band rejection. In order to increase the out-of-band rejection across a wide frequency spectrum, this is achieved with the assistance of triangular shaped capacitive loading at the open-end of the openloop resonators that incorporate "L" shaped slots [10]. The consequence of this is the realisation of harmonic suppression over a very wide frequency bandwidth extending up to $17 \mathrm{GHz}$ as shown in Figure 3.

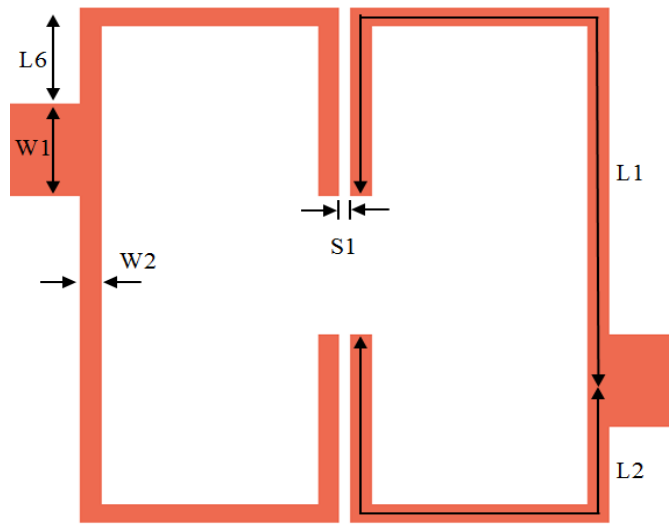

(a)

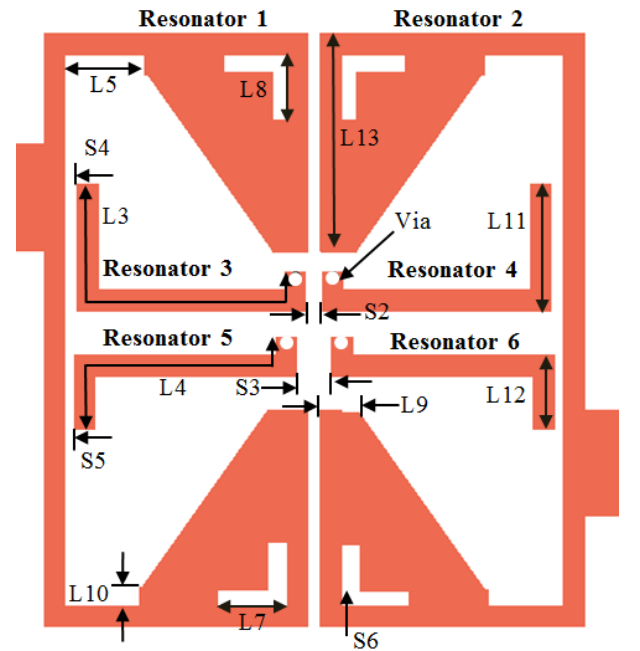

(b)

Fig. 1. (a) The open-loop C-shape resonator and (b) the layout of the proposed filter with embedded resonators.

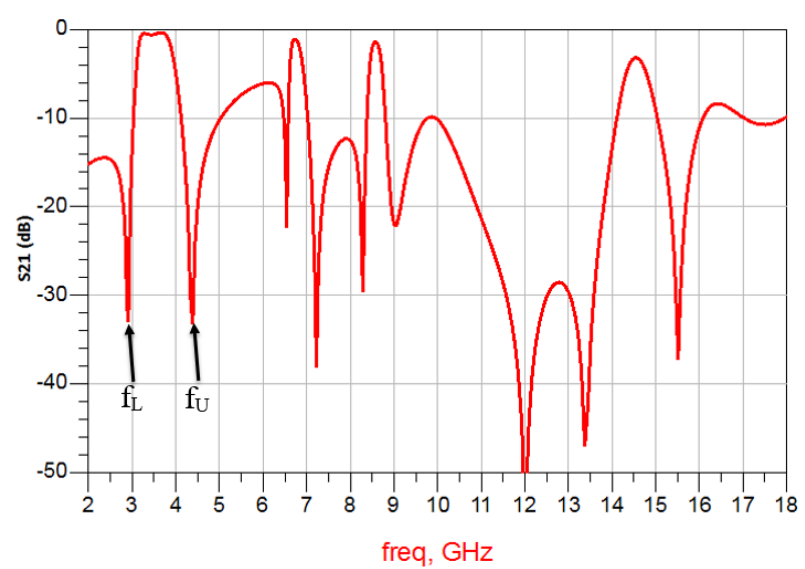

Fig. 2. Frequency response of the Tri-band bandpass filter. 


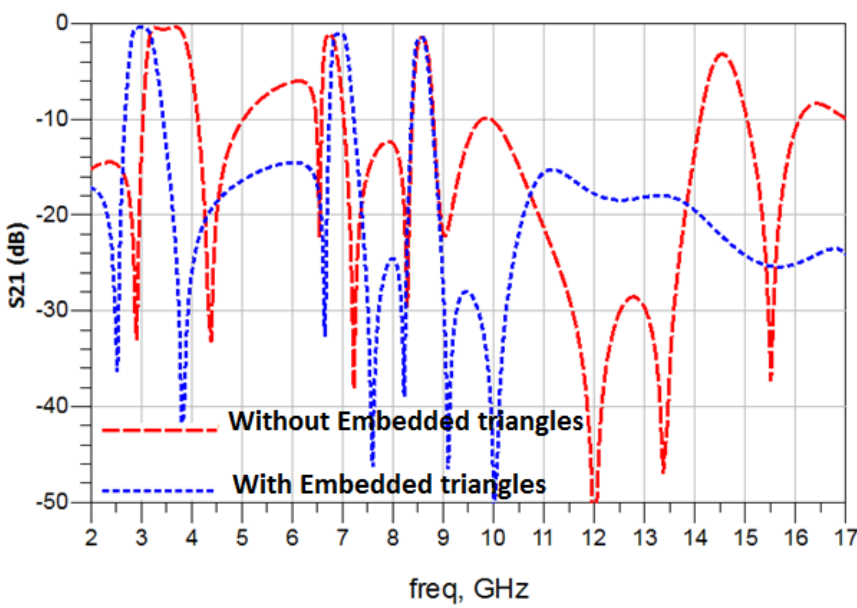

Fig. 3. Simulated insertion-loss response of the proposed filter with and without embedded triangle.

\section{B. Feed and Coupling Scheme}

Figure 4 shows the feed and coupling schemes employed in the filter, where the black and hollow circular nodes represent resonators and source/load, respectively. Figure 4(a) illustrates the feed and coupling scheme at the first resonant frequency $\left(f_{1}\right)$, where resonators 1 and 2 resonate and form a strong electromagnetic coupling with each other and resonate at the first passband $\left(f_{1}\right)$. The corresponding insertion-loss response is shown in Figure 5. In contract, the resonators 3, 4, 5 and 6 are non-resonating at $f_{1}$ however are excited at the second and third passbands. Similarly, Figure 4(b) shows the feed and coupling scheme at the second resonant frequency $\left(f_{2}\right)$, where resonators 3 and 4 resonate at second passband $\left(f_{2}\right)$. The corresponding insertion-loss response is shown in Figure 5. In contract, the resonators 1, 2, 5 and 6 are non-resonating at $f_{2}$. Finally, Figure 4(c) shows the feed and coupling scheme at the third resonant frequency $\left(f_{3}\right)$, where resonators 5 and 6 resonate at third passband $\left(f_{3}\right)$. The corresponding insertion-loss response is shown in Fig. 5. In contract, the resonators 1, 2, 3 and 4 are nonresonating at $f_{3}$, however are excited at other passbands. Source to load coupling is inherently realized as the non-resonating nodes are directly coupled with the resonating nodes.

The intensity of the surface current distributions of the structure at different resonance frequencies are shown in Figure 6. It is clear to see that only the desired resonators are resonating at its associated frequencies, and forming strong EM coupling. The passband bandwidth of the filter depends on the external Qfactor and the coupling coefficient between the resonator pairs. In particular, this depends on the gap (S1, S2, S3, S4 and S5) and the width (W2).

\section{FILTER IMPLEMENTATION}

The design procedure of the tri-band bandpass filter is now described. The initial dimensions of the filter are determined based on the design specifications, in particular the desired passbands frequencies. Then, fine tuning is necessary to achieve the desired response using an appropriate simulation tool.

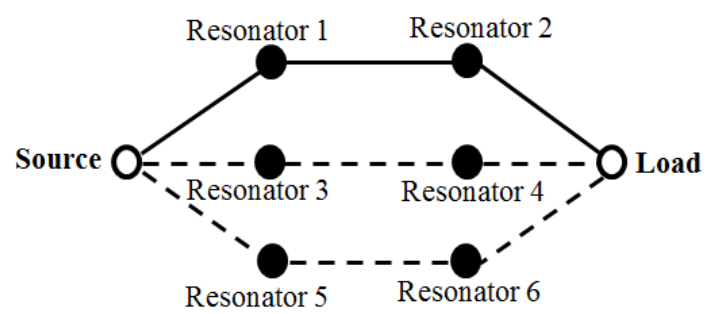

(a)

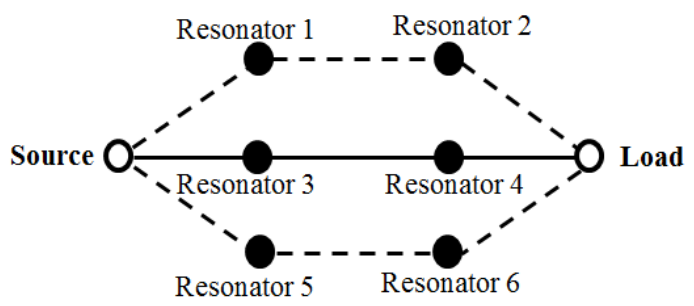

(b)

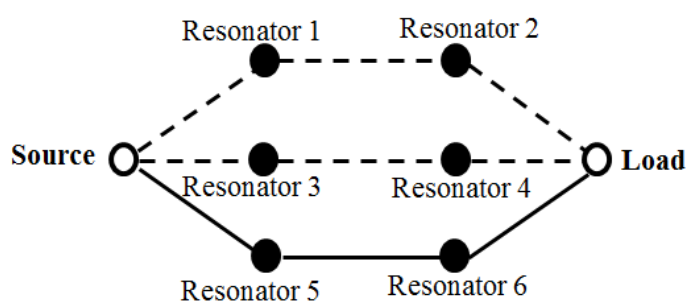

(c)

Fig. 4. Feed and coupling scheme, (a) at the first resonant frequencies, (b) at the second resonant frequencies, and (c) at the third resonant frequency.

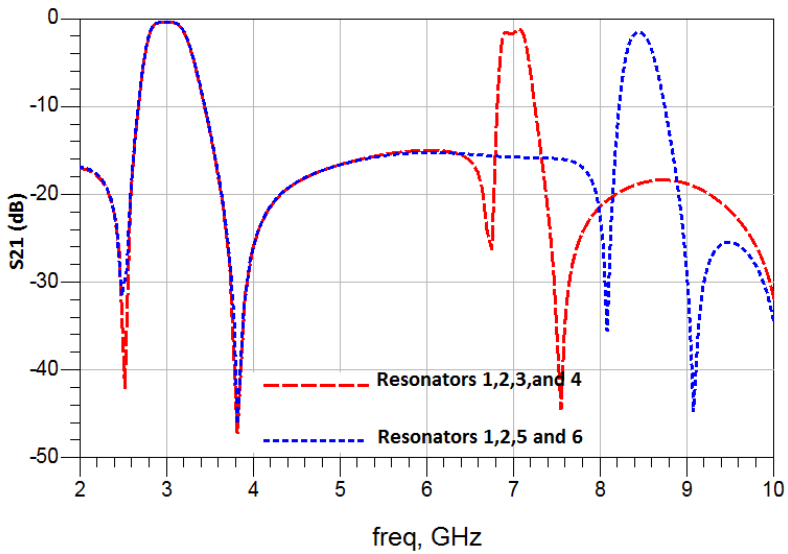

Fig. 5. Frequency response of the quad-band bandpass filter.

Resonator pair 1 and 2 determines the center frequency of the first passbands, the length of the resonators can be calculated from (1), (2) and (3). On the other hand, resonators 3 and 4 determine $f 2$ according to (4), and resonators 5 and 6 determine $f 3$ according to (5). In this design technique the inner resonators have no loading effect over the first passband and over each other, as is evident from Figure 5. Thus, the second and third passband can be controlled individually to any desired 
frequencies, as is evident from Figure 5. In additional, the proposed filter has the ability to be designed as a dual band by excluding any pairs of the inner resonators as shown in Figure 5.

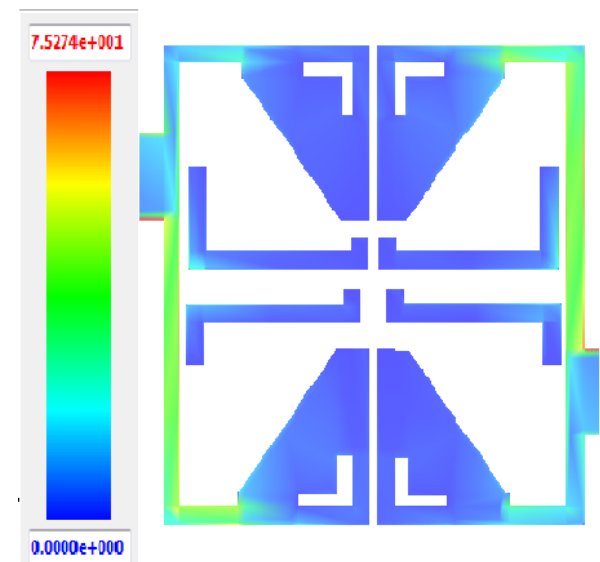

(a)

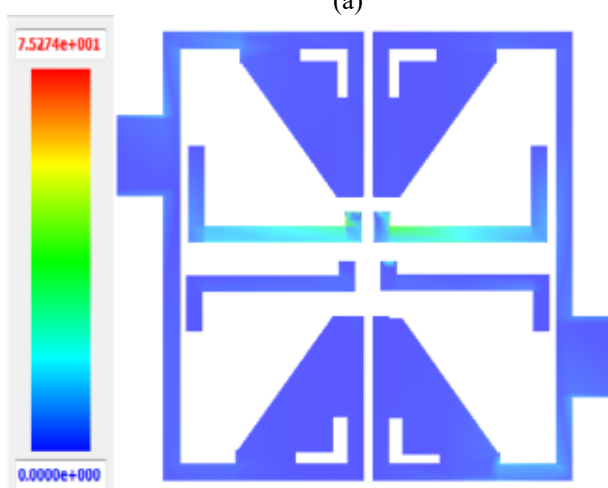

(b)

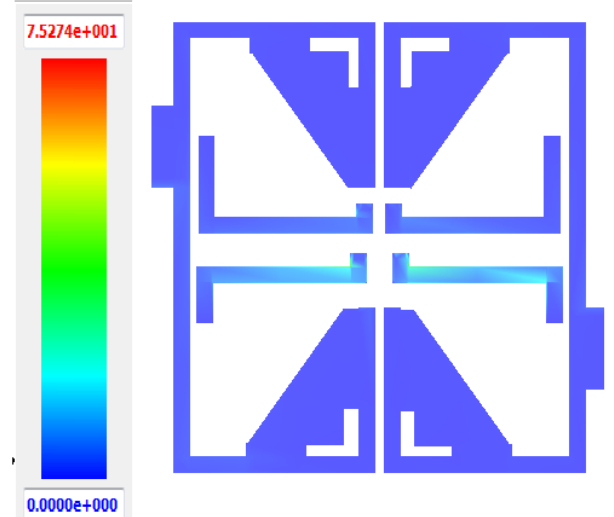

(c)

Fig. 6. The surface current distribution of the proposed filter (a) at the fist $(3 \mathrm{GHz}),(\mathrm{b})$ the second $(6.9 \mathrm{GHz})$ and $(\mathrm{c})$ the third resonance frequency $(8.6$ $\mathrm{GHz}$ ), respectively.

The second step is to determine the required bandwidth for each passband. As mentioned earlier the bandwidth of the filter's passbands depends on the external Q-factor and the coupling-coefficient between resonators. Also, in this design the three pairs of resonators have no coupling effect over each other. Thus, the bandwidth of the three passbands can be determined separately. Coupling-coefficient for the first passband depends on $\mathrm{S}_{1}, \mathrm{~W}_{2}$ and $\mathrm{L}_{13}$, whereas the coupling-coefficient for the second passband depends on $\mathrm{S}_{2}, \mathrm{~W}_{2}$, and $\mathrm{S}_{3}, \mathrm{~W}_{2}$ for the third passband. The external Q-factor is determined by $\mathrm{L}_{11}, \mathrm{~L}_{12}, \mathrm{~W}_{2}$, $\mathrm{S}_{4}$ and $\mathrm{S}_{3}$. By having a smaller gaps (S), a narrower width (W), a longer length $(\mathrm{L})$ will result in a stronger coupling and hence a smaller external Q-factor. On the contrary, the bigger gaps, wider width, a shorter length result in a weaker coupling and hence a higher external Q-factor.

\section{SimUlation RESUlTS}

To demonstrate the feasibility of the proposed concept, a triband filter was designed for fundamental resonant frequencies at $3 / 6.9 / 8.6 \mathrm{GHz}$. The filter was simulated on a substrate with a $0.76 \mathrm{~mm}$-thick and relative dielectric constant 2.17. Following the design methodology in the preceding section the filter's dimensions were determined to be (in millimeters): $\mathrm{L}_{1}=19.5, \mathrm{~L}_{2}$ $=13, \mathrm{~L}_{3}=8, \mathrm{~L}_{4}=6, \mathrm{~L}_{5}=1.6, \mathrm{~L}_{6}=2.5, \mathrm{~L}_{7}=1.4, \mathrm{~L}_{8}=1.3, \mathrm{~L}_{9}=1$, $\mathrm{L}_{10}=0.5, \mathrm{~L}_{11}=2.4, \mathrm{~L}_{12}=1.2, \mathrm{~L}_{13}=5, \mathrm{~W}_{1}=0.5, \mathrm{~S}_{1}=0.23, \mathrm{~S}_{2}=$ $0.34, \mathrm{~S}_{3}=0.74, \mathrm{~S}_{4}=0.25, \mathrm{~S}_{5}=0.23$, and $\mathrm{W}_{2}=2.42$, which corresponds to a characteristic impedance of $50 \Omega$. Simulation was performed using Keysight Technologies' Momentum 3D planar EM simulator based on the method-of-moment (MoM).

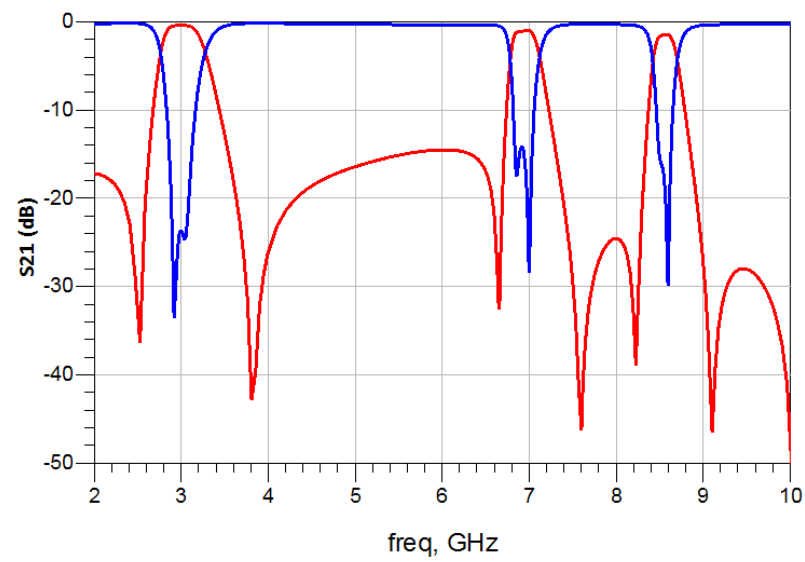

Fig. 7. Simulated narrowband insertion-loss and return-loss response of the proposed filter.

Figures 7 and 8 show the simulation results narrow band and wideband, respectively. The filter has three passband responses centered at 3/6.9/8.6 GHz, with a high out-of-band rejection more than $12 \mathrm{~dB}$. The insertion-losses at the three passbands are less than $0.4 \mathrm{~dB}, 1 \mathrm{~dB}$, and $1.4 \mathrm{~dB}$, respectively. The corresponding return-losses are greater than $24 \mathrm{~dB}, 15 \mathrm{~dB}$ and 16 $\mathrm{dB}$, respectively. The six transmission zeros between the passbands result in the realization of a filter with high selectivity and high isolation between adjacent passbands. As shown in Fig. 8 the filter provides ultra-broadband spurii rejection greater than $15 \mathrm{~dB}$ up to $17 \mathrm{GHz}$.

\section{CONCLUSION}

A highly compact planar Tri-band bandpass filter was presented based on two pairs of $\lambda / 4$ resonators embedded inside the open loop resonators. The proposed technique provides a sufficient degree of freedom to alter the filter's specifications in 
terms of centre frequency and bandwidth. Source to load coupling and the resulting three transmission paths are inherently realized to create transmission zeros, resulting in high selectivity and high isolation between the four passbands. The design methodology has been verified through simulation results. The filter's performance and compact size makes it attractive for application in multiband wireless communication systems.

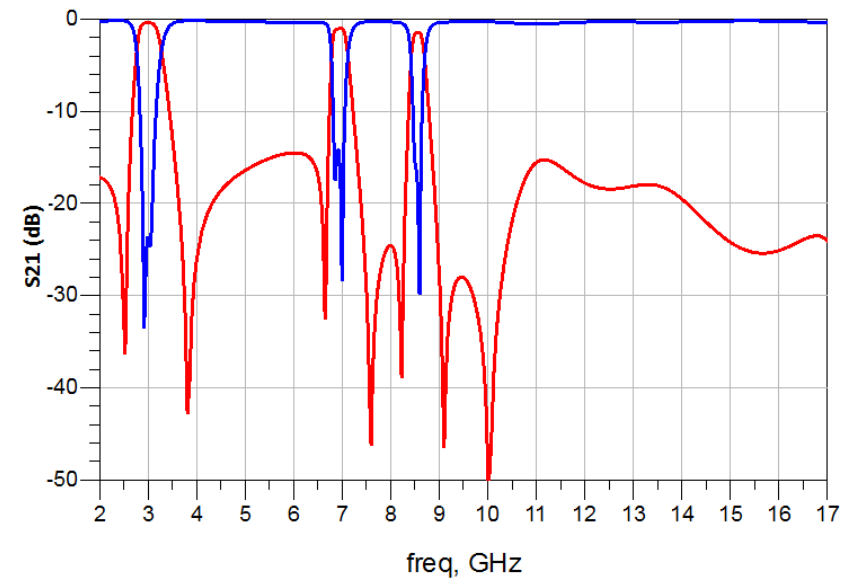

Fig. 8. Simulated wideband insertion-loss and return-loss response of the proposed filter.

\section{REFERENCES}

[1] C. F. Chen, T. Y. Huang, R. B. Wu, "Design of dual- and triple passband filters using alternately cascaded multiband resonators", IEEE Trans. Microw. Theory Tech., Vol. 54, No. 9, pp. 3550-3558, 2006

[2] C. H. Lee, C. I. G. Hsu, H. K. Jhuang, "Design of a new Tri-band microstrip BPF using combined quarter-wavelength SIRs", IEEE Microw. Wire. Compon. Lett., Vol. 16, No. 11, pp. 594-596, 2006

[3] C. I. G. Hsu, C. H. Lee, Y. H. Hsieh, "Tri-band bandpass filter with sharp passband skirts designed using tri-section SIRs", IEEE Microw. Wirel. Compon. Lett., Vol. 18, No. 1, pp. 19-21, 2008

[4] Q. X. Chu, X. M. Lin, "Advanced triple-band bandpass filter using trisection SIR”, Electron. Lett., Vol. 44, No. 4, pp. 295-296, 2008

[5] H. W. Liu, Y. Wang, X. M. Wang, J. H. Lei, W. Y. Xu, "Compact and High selectivity tri-band bandpass filter using multimode steppedimpedance resonator", IEEE Microw. Wireless Compon. Lett., Vol. 23, No. 10 , pp. $536-538,2013$

[6] L. Gao, J. Xiang, Q. Xue, "Novel compact tri-band bandpass filter using multi-stub-loaded resonator", Progress In Electromagnetics Research C, Vol. 50, pp. 139-145, 2014

[7] X. Y. Zhang, L. Gao, Z. Y. Cai, X. L. Zhao, "Novel tri-band bandpass filter using stub-loaded short-ended resonator", Progress In Electromagnetics Research Letters, Vol. 40, pp. 81-92, 2013

[8] Y. Peng, L. Zhang, Y. Leng, J. Guan, "A compact tri-band passband filter based on three embedded bending stub resonators", Progress In Electromagnetics Research Letters, Vol. 37, pp. 189- 197, 2013

[9] M. Farhat, B. S. Virdee, "Dual-Band Bandpass Filter Using Open-Loop Resonators", IEEE Proceedings of Asia-Pacific Microwave Conference, Melbourne, pp. 1054 - 1057, December 5-8, 2011

[10] M. Farhat, B. S. Virdee, K. U. Ahmed "Novel Microstrip Bandpass Filter With Ultra-Wide Stopband", German Microwave Conference, pp. 29-32, March 12-14, 2012 